\title{
BZLF1 Expression of EBV is correlated with PARP1 Regulation on Nasopharyngeal Carcinoma Tissues
}

\author{
Wahyu Nur Laili Fajri', Achmad Rofi'i², Fatchiyah ${ }^{1 *}$ \\ ${ }^{1}$ Biology Departement, Faculty of Mathematics and Natural Sciences, Brawijaya University, Malang, Indonesia \\ ${ }^{2}$ Ulin Hospital, Banjarmasin, Indonesia
}

\begin{abstract}
Nasopharyngeal carcinoma (NPC) is a cancer that arises inside of the nasopharyngeal mucosa and nasopharynx. Epstein Barr Virus (EBV) has been shown to be associated with the development of NPC. Latent Membrane Protein 1 (LMP1) function include to activate BamHI-Z Leftward Reading Frame 1 (BZLF1)-EBV. The infection tissues respond to the expansion of EBV infection by activating Poly(ADP-ribose)Polymerase-1 (PARP1). The objective of this study are to observe the expression of BZLF1 and to determine the PARP1 regulation in NPC tissues. This study used three kind of tissue slides, there are non-keratinizing carcinoma, undifferentiated carcinoma, and polyp. Tissue slides were stained by HE used for characterization of the necrotic cells such as pyknosis cells, karyorrhexsis cells, and karyolysis cells. Tissues slides were analyzed by immunohistochemical (IHC) assays using EBV BZLF1-FITC and anti-PARP1-Rhod. The percentage of necrotic cells and the expression intensity of BZLF1 and PARP1 were analyzed using statistical analysis ( $\mathrm{P}$-value $<0.05)$. This study also used correlation and regression analysis. The research showed that the number of karryorhexis cells higher than pyknosis cells and karyolysis cells in NPC and polyp tissues. The high expression intensity of BZLF1 induced the increase of expression intensity of PARP1, and the expression intensity of BZLF1 and PARP1 are not correlated with percentage of necrotic cell. Interestingly, all tissues showed the increased number of karyolysis cells but the number of pyknosis cells and karryorrhexis cells were remain low. The intensity of expression indicated that the BZLF1 induce PARP1 to repair DNA damage against EBV infection.
\end{abstract}

Keywords: BZLF1, CLSM, EBV, nasopharyngeal carcinoma, PARP1

\section{INTRODUCTION}

NPC is a cancer that arises in the epithelial tissues that covers lining of surface, inner mucosa and crypts of nasopharynx. Features of cancer were included rapid growth of cells, increased cell turn-over, invasive growth, metastasis, vascular or lymphatic channel invasion for malignant lesions [1-2]. EBV is a human herpesvirus, and be a mayor risk for NPC. Most cells and blood of patients have a part of EBV. All herpes viruses have two phases life cycle, involved latency and lytic (reactivation or replication) phase.

EBV in a latent infection is associated with the expression of nine latent proteins. Latent

\footnotetext{
${ }^{*}$ Corresponding author: Fatchiyah

Biology Department, Faculty of Mathematics and Natural Sciences, Brawijaya University, Malang, Indonesia 65145 Email: fatchiya@ub.ac.id
}

membrane Protein 1 (LMP1) is one of the latent proteins, and major EBV oncoprotein. This protein functions include for metastasis, apoptotic resistance, immune modulation and toactivate BamHI-Z Leftward Reading Frame 1 (BZLF1)-EBV, which can inhibit p53 to induce apoptotic resistance, metastasis, and immune modulation. BZLF1 is one of immediate-early (IE) transcription factors, that are responsible for the expression of packing proteins of EBV. BZLF-EBV functions to induce lytic infection in EBV infected cell [3-4].

The infection tissues respond to the expansion of EBV infection by activating Poly (ADP-ribose) Polymerase-1 (PARP1). The synthesis of PARP1 is one of the early DNA damage responses, together with other DNA sensing molecules, such as DNA-PK, ATM and p53 [5]. Over expression of PARP1 estimated from EBV-DNA integrated with host-DNA 
and this may induce a decrease in $\mathrm{NAD}^{+}$and ATP levels which promotes cell dysfunction and cell death by necrosis [6].

Correlation among PARP1, BZLF1-EBV, and necrotic percentage in NPC tissue are still unclear. The objective of this study is to observe the expression of BZLF1 and determine the PARP1 regulation in nasopharyngeal carcinomas tissues.

\section{MATERIALS AND METHODS}

Three kinds of histopathological tissue slides, non-keratinize carcinoma, undifferentiated carcinoma, and polyp was used in this study. All of these tissues slides were collected from Ulin Hospital, Banjarmasin, South Kalimantan. NPC tissues were obtained from biopsy which was done for early prediction of NPC patients. Methods and protocol of this study was approved by the Ethical Research Commission of Brawijaya University (Indonesia).

The number of necrotic cells and the expression intensity of BZLF1 and PARP1 of each histopathological tissue slides were observed. This study used two kind of staining methods, hematoxylin eosin (HE) and immunohistochemical (IHC) assays. HE staining was conducted at Ulin Hospital, South Kalimantan. Histological slides were stained by HE used for characterization of the necrotic cells such as pyknosis (nuclear shrinkage) cells, karyorrhexsis (nuclear fragmentation) cells, and karyolysis (nuclear fading) cells. The necrotic cells observed using a BX-53 microscope (Olympus) with CellSens Standard software at the Biosains Laboratory, Brawijaya University, Malang.

Tissues slides were analyzed by IHC, modified from [7] by using EBV-BZLF1 Mouse anti-EBV monoclonal antibody (Lifespan Biosciences) (1:1000) against Goat antimouse IgG-FITC (Santa Cruz Biotechnology) (1:1500) and anti-PARP1 antibody (MC-10) $(1: 1500)$ against Goat anti-mouse $\operatorname{IgG}$ labeled Rhodamin (Santa Cruz Biotechnology) (1:1500).

The expression intensity of BZLF1 and PARP1 from fluorescent colour was observed by Confocal Laser Scanning Microscope (Olympus) with Olympus FluoView software version $1.7 \alpha$ at the Central Laboratory of Life Sciences (LSIH). Visualization of expression of BZLF1 and PARP1 was performed on three fields of view of each slide. Measurement of the expression intensity of each field of view is taken from 5 points. Observations and measurements take on the location of the expression of BZLF1 and PARP1.

The percentage of necrotic cells and expression intensity of BZLF1 and PARP1 were analyzed using SPSS 16.0 by one-way ANOVA test $(\mathrm{P}$-value $<0.05)$. This study used correlation analysis to test the statistical significance of association between variables, and regression analysis to identify the effect of each variable.

\section{RESULTS AND DISCUSSION}

The result of this study showed that karyorrhexsis cells more commonly found than pyknosis cells and karyolysis cells. All tissues (non keratinize carcinoma, undifferentiated carcinoma, and polyp) showed the increased number of karyolysis cells, but the number of pyknosis cells and karryorrhexis cells were remain low (Fig. 1).

The high percentage of karyorrhexsis cells in all tissues indicates that the nucleus was damage and PARP1 failed to repair damaged cells, therefore the tissue target has a mechanism of cell death by necrosis.

This result was similar with previous research by Cepeda et al. [6] which states that DNA damage will cause a very large excess of PARP1 expression, this may induce a decrease in NAD + and ATP levels which lead to cell dysfunction and even cause cell death by necrosis.

The results of this study showed that the expression intensity of BZLF1 and PARP1 in polyp tissue tends to be lower than the non keratinize carcinoma, undifferentiated carcinoma. The expression intensity of BZLF1 and PARP1 at polyp tissue were remain low (Fig. 2 (supplement 1)). Nevertheless, the results of statistical analysis showed that the intensity of expression of BZLF1 and PARP1 was not significantly different in polyp, non keratinize carcinoma and undifferentiated carcinoma. The high intensity of expression of BZLF1-NPC showed that the infection has reached the stage of EBV lytic phase.

BZLF1 is a protein that plays a role in the lytic phase of EBV [3]. When the initially of EBV infection, LMP1 has been started to enable exposure to BZLF1. In addition, over expression of BZLF1 in EBV-infected cells is sufficient to initiate the conversion of latent infection to lytic / productive infection [8]. 

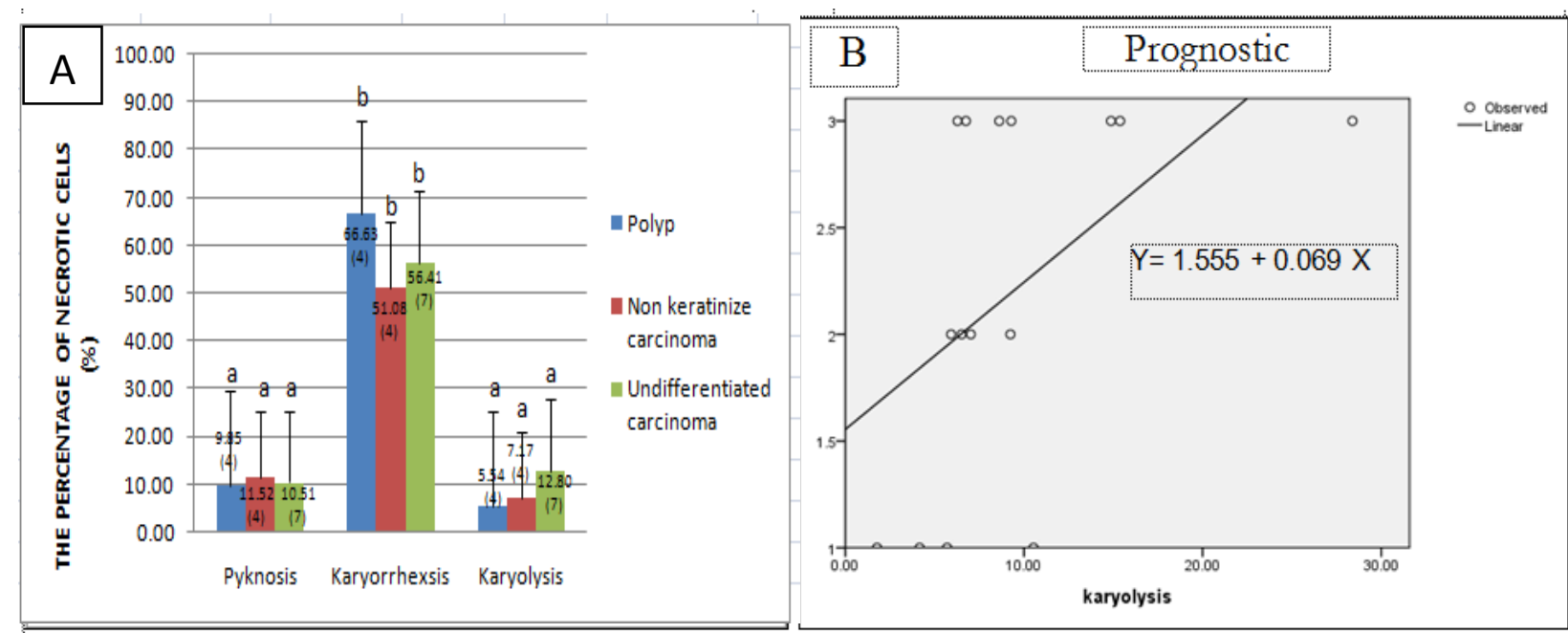

Figure 1. The percentages of necrotic cells (pyknosis cells, karyorrhexsis cells, and karyolysis cells) (A), and the result of regression analysis of karyolysis cells (B).

BZLF1 is a transcription factor immediateearly (IE) than BRLF1, which is responsible for the expression of proteins required in the form capable of infecting virus [4]. Although BZLF1 was essentially required in the replication of oriLyt, a mechanism is also assisted by another protein, BMRF1 are required in the viral polymerase [8]. On polyp tissue the levels of EBV infection were low, presumably because it has not reached the stage of EBV lytic infection, the expression intensity of BZLF1 in polyp is lower than in NPC. Gelardi [9] found that the nasal polyp is a benign tumor, while NPC is malignant tumors.

The higher value of expression intensity in PARP1-NPC than PARP1 in polyps was due to infection of EBV in NPC conditions will activate PARP1 which is protection of cells against DNA damage. PARP1 is involved in initiation of DNA damage response mechanisms, including DNA repair, cell cycle arrest and apoptosis [10].

A number of PARP1 inductions are included gamma radiation, $\mathrm{TNF} \alpha$, alkylating agents, $\mathrm{UVB}$, and free radicals. After the cells exposed to induction agents of PARP1, the PARP1 will bound directly to DNA damage and have auto modification thus forming polymer poly (ADPRibose) long, branched at the target protein, using the substrate $\mathrm{NAD}^{+}$, which affects the depletion of $\mathrm{NAD}^{+}$intracellular [11].

PARP1 is the first cellular protein identified in the mechanism of apoptosis [5]. Activation of caspase-3 (ACA-3) break down proteins, including poly (ADP Ribose) polymerase-1 (PARP1) is important in DNA repair, causing by apoptosis. Over activation of PARP1 led to deplet- ion of $\mathrm{NAD}^{+}$and ATP, and causing necrosis [11].

The severity of disease in polyp is lower than in the NPC. It was shown that the expression intensity of PARP1 in polyp lower than in NPC. The expression intensity of BZLF1 tends to be lower than PARP1. This condition occurred because in the normal condition PARP1 were synthesized even in small amounts, whereas BZLF1 will be expressed when the infection of EBV reaches to the lytic stage.

PARP1 is responsible for post-translational modification of proteins in response to many endogenous and environmental genotoxic agents. PARP and poly (ADP-ribosyl)ation to be something important in the regulation of many cellular processes such as DNA repair, cell death, chromatin functions and genomic stability [5].

\section{CONCLUSIONS}

The result of this study showed that the highest number of necrotic cells was karryorhexsis cells in all tissues. Interestingly, all tissues (non keratinize carcinoma, undifferentiated carcinoma, and polyp) showed that the increased number of karyolysis cells, but the number of pyknosis cells and karryorrhexis cells were remain low. The high expression intensity of BZLF1 induced the increasing of expression intensity of PARP1, but not correlated with percentage of necrotic cell. The intensity of expression indicated that the BZLF1 induce PARP1 to repair DNA damage against EBV infection. 


\section{ACKNOWLEDGEMENT}

We thanks to Dr. Sri Widyarti and Dr. Estri Laras Arumingtyas from Biology Department, Brawijaya University for reviewing this paper, and also to Praba Sanjaya Putra, S. Si for assisting to use CLSM analysis.

\section{REFERENCES}

1. Jain, D, Parkash V, Li M, Gill J, Crouch J, Howe G, Tallini G (2000) Epstein-Barr virus RNA detection and glandular differentiation in nasopharyngeal carcinoma: report of 2 cases. Archives of pathology \& laboratory medicine, 124 (9): 1369-72.

2. Idikio, HA (2011) Human Cancer Classification: A systems biology-based model integrating morphology, cancer stem cells, proteomics, and genomics. Cancer. 107-115.

3. Ning S (2011) Innate immune modulation in EBV infection. Herpesviridae. 2 (1): 1.

4. Zhang, QIN, Gutsch D, Kenney S, Carolina, N, Hill C (1994) Functional and physical interaction between $\mathrm{p} 53$ and BZLF1: Implications for epstein-barr virus latency. Microbiology. 14 (3): 1929-1938.

5. Herceg, Z, Wang ZQ (2001) Functions of poly (ADP-ribose) polymerase (PARP) in DNA repair, genomic integrity, and cell death. Mutation Research. 477: 97-110.

6. Cepeda, V, Fuertes MA, Castilla J, Alonso C, Quevedo C, Soto M, Perez JM (2006) Poly (ADP-Ribose) Polymerase-1 (PARP-1) inhibitor in cancer chemotherapy. Recent Patens on AntiCancer Drug Discovery. 1 (1): 39-53.

7. Bancroft, JD, Gamble M (2002) Theory and practice of histological techniques. Churchill Living Stone. Elsevier. 105: 121.

8. Zhang, QIN, Hong YU, Dorsky D, Holleyguthrie E, Zalani S, Elshiekh NALY, Kiehl A, Le T, Kenney S (1996) Functional and physical interactions between the epstein-barr virus (EBV) proteins BZLF1 and BMRF1 : Effects on EBV transcription and lytic replication. Microbiology. 70 (8): 5131-5142.

9. Gelardi, M, Marilena T, Michele C, Gaspare B, Maria LF, Agata C, Maria AC, Pasquale C. (2006) Epstein-barr virus induced cellular changes in nasal mucosa. Journal of Virology, 3 (6): 1-6.

10. Masutani, M, Nozaki T, Nishiyama E, Tachi Y, Suzuki H, Nakagama H, Wakabayashi K (1999) Function of poly (ADP-ribose) polymerase in response to DNA damage: Gene-disruption study in mice. Molecular and Cellular Biochemistry. 149-152.

11. Sairanen, T, Szepesi R, Saksi J, Paetau A, Lindsberg PJ (2009) Neuronal caspase-3 and PARP-1 correlate differentially with apoptosis and necrosis in ischemic human stroke. Acta Neuropathologica. 541-552. 


\section{Supplement 1}

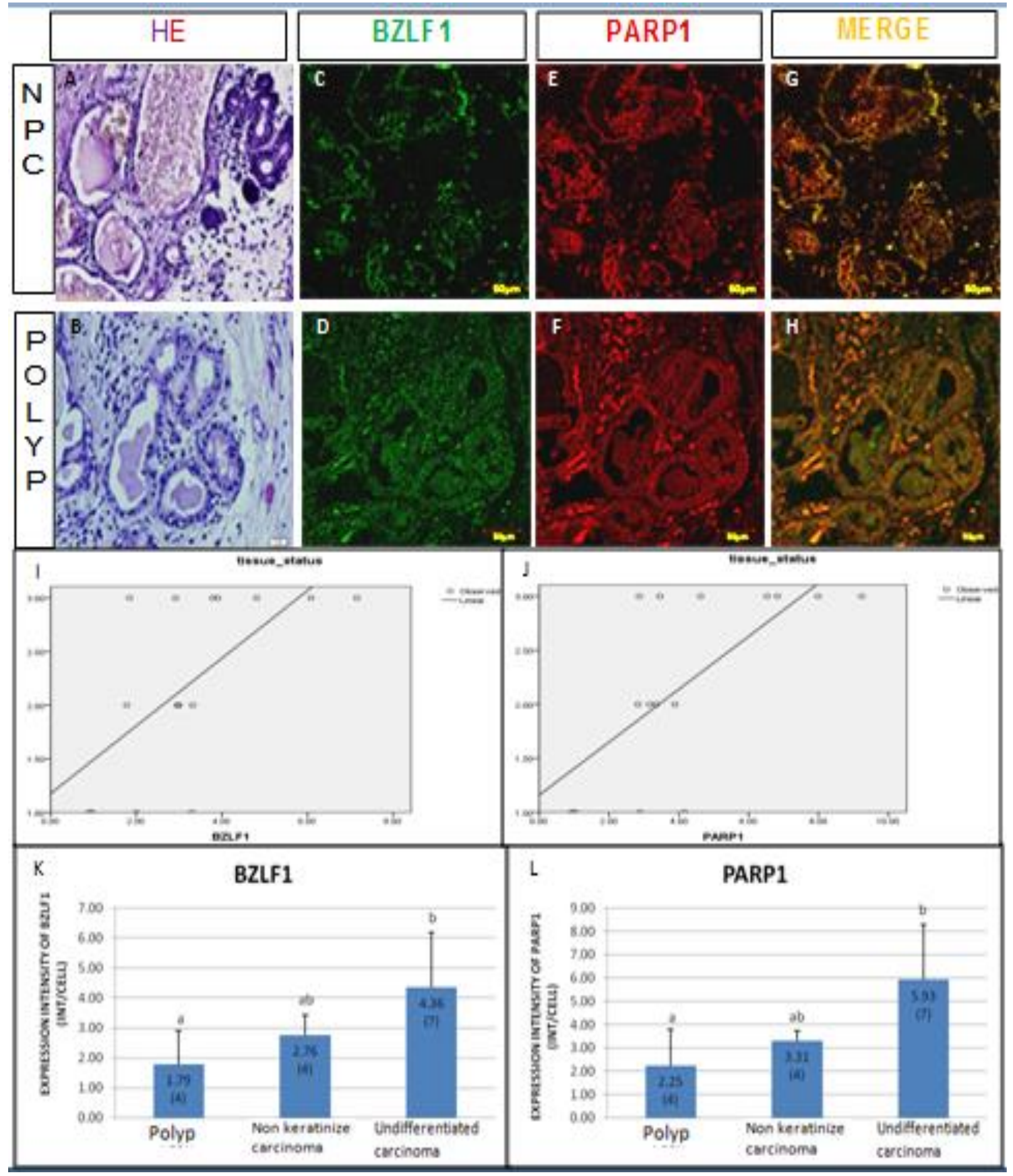

Figure 2. Hematoxylin-eosin-stained slides (A for NPC and B for polyp). IHC result of BZLF1 (C and D), PARP1 (E and F), merge (G and H) (magnification 400x). Regression of BZLF1 (I) and PARP1 (J). The expression intensity of BZLF1 (K) and PARP1 (L) in polyp, non-keratinize carcinoma, and undifferentiated carcinoma 